\title{
Modelo de prevención del maltrato infantil para atención primaria
}

\author{
Prevention model in primary care for child maltreatment
}

Dubowitz H y col. Pediatrics.2009; 123;858-864

\section{Objetivo}

Implementar un modelo de atención para prevenir el maltrato infantil a través de la detección de sus factores de riesgo.

\section{Diseño, lugar y pacientes}

Ensayo controlado y aleatorizado llevado a cabo en un Hospital Universitario de Baltimore, Maryland, Estados Unidos.

Fueron seguidos durante tres años y cinco meses 729 padres angloparlantes de niños menores de cinco años que llevaban a sus hijos al control de salud, que no tuvieran otro hermano en el estudio y que sus hijos no estuvieran a cargo de un cuidador.

\section{Intervención y medición de resultados principales}

Los padres con sus hijos fueron aleatorizados a dos grupos (intervención y control) de acuerdo al día que consultaban: dos días a la semana se aplicaba la intervención y otros dos días, los cuidados estándares.

La intervención constó de un modelo de atención (en inglés: SEEK) que incluye:

1) Dos medias jornadas de entrenamiento a los médicos residentes (MR) para detectar factores de riesgo (FR) como depresión materna, abuso de sustancias, estrés, parejas violentas e implementación de castigos, reforzando el entrenamiento cada seis meses.

2) La solicitud a los padres de que antes de entrar al control de salud de su hijo contesten en la sala de espera un cuestionario auto-administrado para el rastreo de factores de riesgo de maltrato. Ver el cuadro 1.

3) La provisión a los MR de manuales con información sobre recursos locales para el abordaje y la prevención de situación de maltrato y de folletos con información comprensible para los padres.

4) La disponibilidad de una trabajadora social.

Cuadro 1: cuestionario de rastreo de maltrato para los padres.

Querido padre o tutor: ser un padre no es fácil. Queremos ayudar a las familias a crear un ambiente seguro para sus hijos y por eso estamos haciendo a todos los padres estas preguntas. Por favor conteste estas preguntas sobre la base de lo que ocurre con su hijo que hoy viene al control de salud. Son sobre temas que afectan a toda la familia. Si existe algún problema, vamos a tratar de ayudarlos.

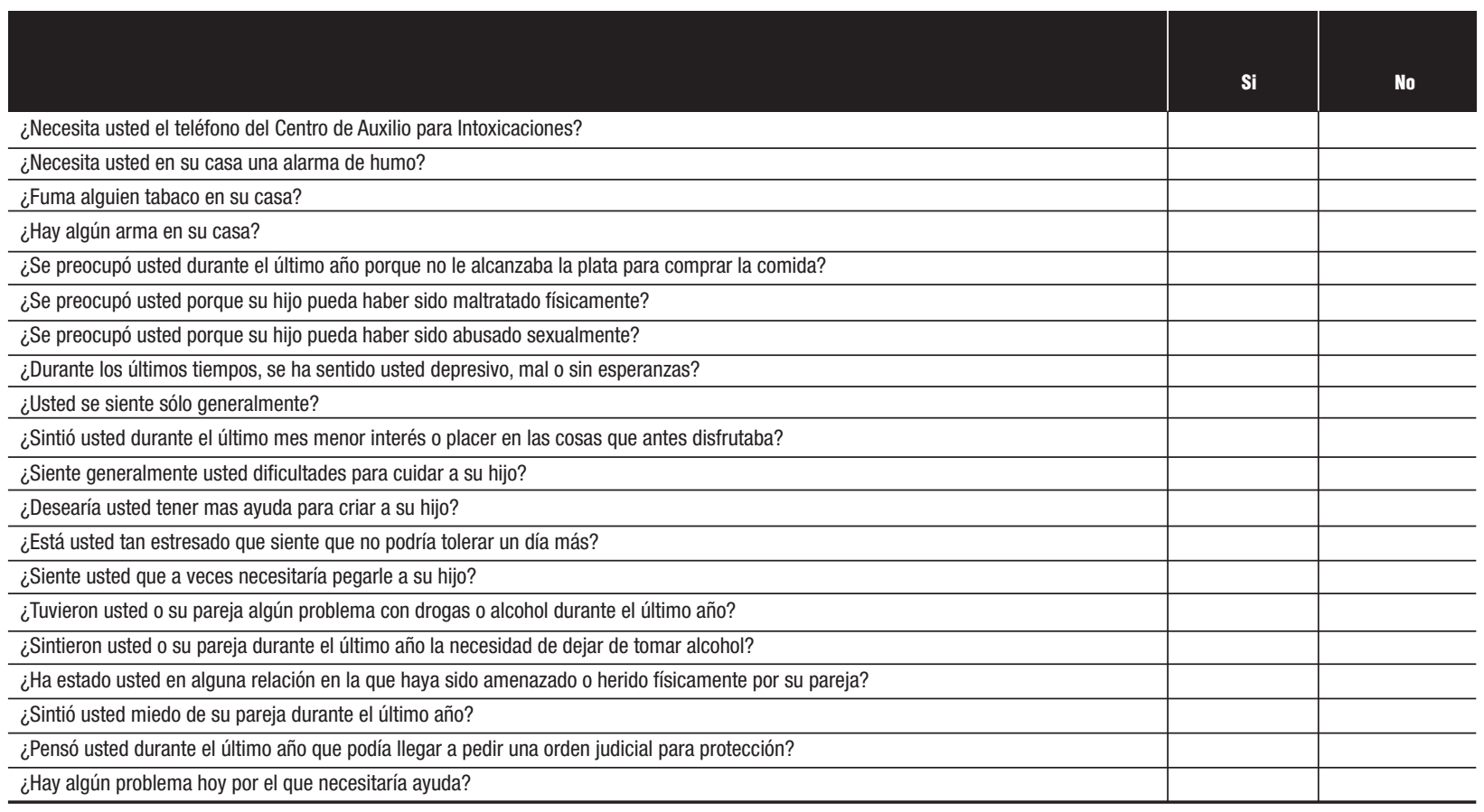

Por favor entréguele este formulario al médico o a la enfermera que usted vea hoy. Muchas gracias.

La traducción que hemos incluido en EVIDENCIA fue realizada por los autores del comentario. El cuestionario original es en inglés, fue publicado por Dubowitz y col. y no existe todavía una versión para su uso en castellano. Dubowitz y col. lo validaron en inglés en una muestra de 200 madres con un $11 \%$ de prevalencia de violencia doméstica. Para determinar las características operativas del grupo de preguntas que rastrean violencia doméstica habían utilizado como prueba de referencia el extenso cuestionario de 76 items "Revised Conflict Tactics Scale". La pregunta " ¿Ha estado usted en alguna relación en la que haya sido amenazado o herido físicamente por su pareja?" fue la de mejor desempeño, con una sensibilidad de $29 \%$, una especificidad de $92 \%$, un valor predictivo positivo de $41 \%$, un valor predictivo negativo de $88 \%$, un coeficiente de probabilidad (en inglés LR) positivo de 3,8 y un LR negativo de 0,77 .

Se midió la incidencia de abuso y descuido en ambos grupos, obtenida a través de tres fuentes de datos:
1) los registros del Servicio de Protección al Menor (CPS); 2) la revisión de historias clínicas; 3) cuestionarios para padres con escalas de disciplina y castigos.

El análisis fue por intención de tratar. 


\section{Resultados}

Se resumen en la tabla 1.

Tabla 1: resultados primarios en ambos grupos.

\begin{tabular}{l|c|c|c} 
& $\begin{array}{c}\text { Grupo } \\
\text { intervención }\end{array}$ & $\begin{array}{c}\text { Grupo } \\
\text { control }\end{array}$ & Odds ratio \\
\hline Al menos una denuncia en el Sistema de Protección de Menores & $41(13,3 \%)$ & $48(19,2 \%)$ & 1,5 \\
\hline Ausencia al control de salud & $4,6 \%$ & $8,4 \%$ & - \\
\hline Retardo en las inmunizaciones & $3,3 \%$ & 0,03 & - \\
\hline Auto-reporte de violencia severa & $0,11(0,75)$ & 0,05 & - \\
\hline
\end{tabular}

Conclusiones

La asignación a la intervención preventiva "SEEK" se asocio con una disminución de la incidencia de abuso y descuido.

Palabras claves: maltrato infantil, prevención, atención primaria de la salud. Keywords: child abuse, prevention, primary care atention. Fuentes de financiamiento: no referida.

\section{Comentario}

Los servicios de Atención Primaria -especialmente los que intervienen en el área comunitaria- tienen un papel destacado en la prevención del maltrato infantil ya que suelen ser los más accesibles -a veces en forma normalizada y generalizada- para las familias durante la edad en que el niño es especialmente vulnerable. En este sentido, los profesionales que trabajan en esta área (ej. médicos de familia o pediatras entrenados en maltrato) se encuentran en una posición favorable para detectar situaciones de riesgo y colaborar en la intervención protectora. Por ejemplo, la expansión del marco de referencia del médico y su fuerte alianza terapéutica con distintos miembros de la familia contribuyen a que visualice e intervenga sobre el problema del niño en el contexto del fortalecimiento familiar. Podemos decir que todo enfoque familiar se basa en la colaboración con la familia con el objetivo de ayudar a los padres a incorporar metas de pareja o de crianza y/o si fuera necesario, métodos parentales alternativos. El estudio de Dubowitz y col. que hemos resumido muestra un modelo de cuidado preventivo cuya virtud es la de minimizar la intervención, evitando que sea visualizada como intrusiva o controladora, disminuyendo así la resistencia al cambio y facilitando la revisión de las prácticas parentales. Ayuda a los padres a ubicarse en una meta-posición en relación a sus propios patrones interacciónales. Ese meta-nivel desde el cual se observan los empuja "a proteger" más que "a protegerse" y a reconocer no solo ciertas necesidades básicas de sus hijos, sino también las propias. Si aparecen interacciones abusivas a nivel familiar podemos pensar que existen fronteras interpersonales poco claras y fallas en la resolución de conflictos. Cuando, como en este estudio, priman las familias uniparentales -en las que un adulto hace toda la tarea- la sobrecarga y la desorganización pueden estar presentes. Esta parentalidad requiere pues, mucho más foco y apoyo para prevenir la conducta autoritaria o la insuficiente atención a los hijos.

Respecto de su metodología, vale destacar la fragilidad de la forma de aleatorización de esta investigación, ya que es difícil que la este tipo de asignación -de acuerdo al día de la semana en que era hecha la consulta- se mantenga en el tiempo sin que exista contaminación* entre los grupos y/o un sesgo de selección* de las familias más graves hacia el grupo intervención, lo que tendería a diluir el hipotético efecto preventivo de la misma. Por ejemplo, es probable que a medida que fue avanzando el reclutamiento de familias en el estudio, el grupo intervención haya ido captando cada vez familias de mayor riesgo. Por ejemplo y si le tenían confianza a la eficacia de la intervención, los médicos de guardia pudieron tener la tentación de recomendarle a las familias que consideran de riesgo, que consulten para el seguimiento el martes o el jueves, si es que esos hubieran sido los días en que se ponía en práctica el programa SEEK. Respecto de las características basales de los pacientes, en su mayoría eran similares, con una edad algo menor de los niños y menor cantidad de habitantes por hogar en el grupo "intervención". La medición de los resultados a través del reporte de las historias clínicas y de los registros de protección de menores otorga seriedad al estudio. Sin embargo, no consideramos confiable la medición de los castigos a través del auto reporte de los padres. Recordemos que se está queriendo medir "violencia", lo que no suele ser reconocido voluntariamente con facilidad; por lo que intuimos un importante sub-registro en esta variable.

\section{Conclusiones de los comentadores}

El maltrato es un indicador de la calidad de vida de la familia. En este sentido, el cuestionario, diseñado para identificar a familias de riesgo aparece como un eficaz instrumento para su detección. El profesional de atención primaria debe estar entrenado para recomendar intervenciones en estos grupos. Sin embargo, vale destacar que las estrategias de prevención del maltrato requieren de todos los recursos de la sociedad y no solo de los sanitarios.

Pedro Herscovici [ TESIS Instituto de Terapias Sistémicas ] pedrohersco@fibertel.com.ar

Ver glosario*

Yamila De Vas [ Servicio de Medicina Familiar de Hospital Italiano de Buenos Aires ] yamila.devas@ hospitalitaliano.org.ar

Recibido el 15/05/09 y aceptado el 02/06/09.

Herscovici P y De Vas Y. Modelo de prevención del maltrato infantil para atención primaria. Evid Act Pract Ambul. 12(3) 88-89 Jul-Sep 2009. Comentado de: Dubowitz H y col. Pediatric Primary Care to Help Prevent Child Maltreatment: The Safe Environment for Every Kid (SEEK) Model. Pediatrics.2009; 123; 858-864. PMID: 19255014.

Disponible bajo suscripción en URL: http://pediatrics.aappublications.org/cgi/content/full/123/3/858 (último acceso 26/05/09).

\section{Referencia}

1.Dubowitz H y col. Screening for depression in an urban pediatric primary care clinic. Pediatrics.2007; 119(3):435-443.

Disponible en URL: http://www.pediatrics.org/cgi/content/full/119/3/435(último acceso 26/05/09).

2. Dubowitz H y col. Screening for Intimate Partner Violence in a Pediatric Primary Care Clinic. Pediatrics 2008;121;e85-e91.

Disponible en URL: http://www.pediatrics.org/cgi/content/full/121/1/e85(último acceso 26/05/09) 